\title{
An Active Hand-Held Instrument for Enhanced Microsurgical Accuracy
}

\author{
Wei Tech Ang, Cameron N. Riviere, and Pradeep K. Khosla
}

The Robotics Institute, Carnegie Mellon University, Pittsburgh, PA 15213, USA

\begin{abstract}
This paper presents the first prototype of an active hand-held instrument to sense and compensate physiological tremor and other unwanted movement during vitreoretinal microsurgery. The instrument incorporates six inertial sensors (three accelerometers and three rate gyros) to detect motion of the handle. The movement of the instrument tip in three dimensions is then obtained using appropriate kinematic calculations. The motion captured is processed to discriminate between desired and undesired components of motion. Tremor canceling will be implemented via the weighted-frequency Fourier linear combiner (WFLC) algorithm, and compensation of nontremorous erroneous motion via an experimental neural-network technique. The instrument tip is attached to a three-degree-of-freedom parallel manipulator, actuated by three piezoelectric stacks. The actuators move the tool tip in opposition to the motion of the tremor or other erroneous motion, thereby suppressing the error. Experimental results show that the prototype is able to follow one-dimensional and three-dimensional trajectories with rms error of 2.5 $\mu \mathrm{m}$ and $11.2 \mu \mathrm{m}$ respectively.
\end{abstract}

\section{Introduction}

Human limitations in positioning accuracy during micromanipulation hamper microsurgical performance. They make some procedures difficult, and some desired procedures impossible. These limitations are due primarily to small involuntary movements that are inherent in hand motion. Involuntary movement and the resulting imprecision have long been a matter of concern in microsurgery [1], and perhaps nowhere more so than in vitreoretinal microsurgery, in which manual imprecision limits both what can be done, and how well it can be done [2]. One example is the treatment of retinal vein occlusions by intraocular cannulation, a procedure that is either extremely difficult or impossible with the bare hands [3,4]. For this and other procedures, there is some degree of consensus among vitreoretinal microsurgeons that instrument-tip positioning accuracy of $10 \mu \mathrm{m}$ is desirable [2]. This would represent an order-of-magnitude or better improvement over the capabilities of unassisted microsurgeons [5].

The most familiar type of involuntary or erroneous movement affecting microsurgery is physiological tremor [6]. Tremor is defined as any involuntary, approximately rhythmic, and roughly sinusoidal movement [7]. Physiological tremor is a type of tremor that is inherent in the movement of healthy subjects. The component of physiological tremor that is generally evident in vitreoretinal microsurgery is what Elble and Koller [7] call the "neurogenic" component: an oscillation at $8-12 \mathrm{~Hz}$ whose frequency is independent of the mechanical properties of the hand and arm. The resulting tool tip oscillation during vitreoretinal microsurgery is typically $50 \mu \mathrm{m}$ peak-to-peak (p-p) or greater $[8,9]$. 
There are other significant components of erroneous motion in microsurgery. Measurements of the hand motion of surgeons have shown the significance of nontremorous components of motion such as jerk (i.e., normal myoclonus), drift, and certain vaguely defined and poorly understood low-frequency undesired components [5]. These components are often larger than physiological tremor [5]. Therefore, in working toward positioning accuracy of $10 \mu \mathrm{m}$, suppressing physiological tremor is necessary but not sufficient.

Engineering approaches to the problem of increasing accuracy in microsurgery have been various. Several efforts have been based on traditional telerobotic approaches $[9,10]$, in which filtering can be inserted between master and slave manipulators. Motion scaling can also be implemented in such systems. Taylor et al. have followed a "steady hand" approach, in which a robot and a surgeon directly manipulate the same tool [11], with the robot having high stiffness, and moving along with only those components of the manual input force that are deemed desirable. While such a system cannot scale input motion, it has advantages in terms of cost and likelihood of user acceptance. In order to further reduce cost, and to maximize ease of use, user acceptance, and compatibility with current surgical practice, the present authors are implementing accuracy enhancement within a completely hand-held tool, seeking to keep the instrument size and weight as close as possible to those of existing passive instruments. In such a device, simple lowpass filtering is inadequate; instead, the system must generate a specific estimate of undesired motion, so that the tool can deflect its own tip by an equal but opposite amount, and must do so with no time delay, so that the deflection will be in phase with the erroneous motion to be compensated. This paper presents the design, implementation, and preliminary experimental results of the first prototype of Micron, an active hand-held instrument for compensation of physiological tremor and other position errors in microsurgery. While the initial design is geared toward vitreoretinal microsurgery, the principles involved are general.

\section{Design and Implementation}

\subsection{System Requirements}

A typical vitreoretinal microsurgical instrument is 7.5 to $15 \mathrm{~cm}$ long and 1.0 to $1.5 \mathrm{~cm}$ in diameter. The intraocular shaft, roughly $3 \mathrm{~cm}$ long, with outer diameter of about 1 $\mathrm{mm}$, is fitted with an end-effector (e.g., pick, forceps, scissor). Access to the interior of the eye is made through a sclerotomy. To be practical, an active microsurgical instrument should be of similar size to existing passive instruments. Weight must be minimized in order to avoid fatigue.

Unlike a telerobotic system, in which unwanted motion is suppressed by filtering it out, an active instrument must actually replicate the unwanted motion, generating an equal but opposite tip displacement. To achieve active error compensation, the instrument must sense its motion, distinguish desired from undesired motion in real time, and deflect its tip to nullify the undesired motion. The sensing and actuation bandwidth must be greater than $12 \mathrm{~Hz}$, in order to allow canceling of physiological tremor, which is nominally $8-12 \mathrm{~Hz}$ [7]. In active compensation, the compensating tip deflection must be in phase with the erroneous motion, so error estimation algorithms must operate without time delay or lag. A range of tip motion of $100 \mu \mathrm{m}$ 
in each of the three coordinate directions suffices for tremor canceling [9]. For canceling of certain non-tremorous types of erroneous motion, a range of motion greater than $300 \mu \mathrm{m}$ is needed [5].

\subsection{System Overview}

A picture of our current system is shown in Fig. 1(a). The instrument weighs $170 \mathrm{~g}$, measures $210 \mathrm{~mm}$ in length (including the $30 \mathrm{~mm}$ intraocular shaft) and has an average diameter of $22 \mathrm{~mm}$. The narrowed section of the handle near the tip is contoured as an aid to securely grasping the instrument.

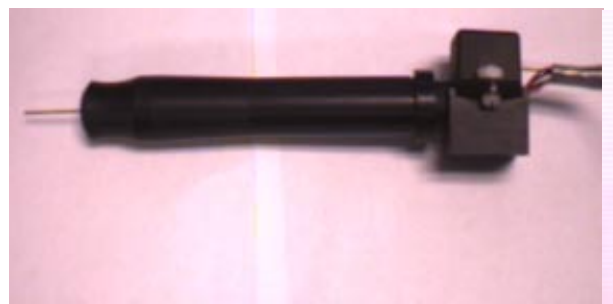

(a)

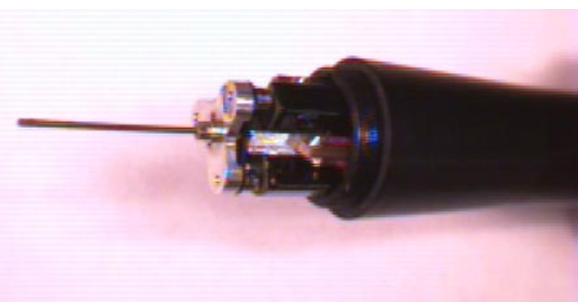

(b)

Fig. 1. Micron. (a) The entire active microsurgical instrument. (b) Close-up of the tip manipulator, with cover removed

An overview of the complete system is presented in Fig. 2. The current system controls the piezoelectric actuators in open loop. In the future, strain gauges will be added to sense the deflection of the actuators in order to provide closed-loop control. This feedback loop is indicated by dotted arrows in Fig. 2.

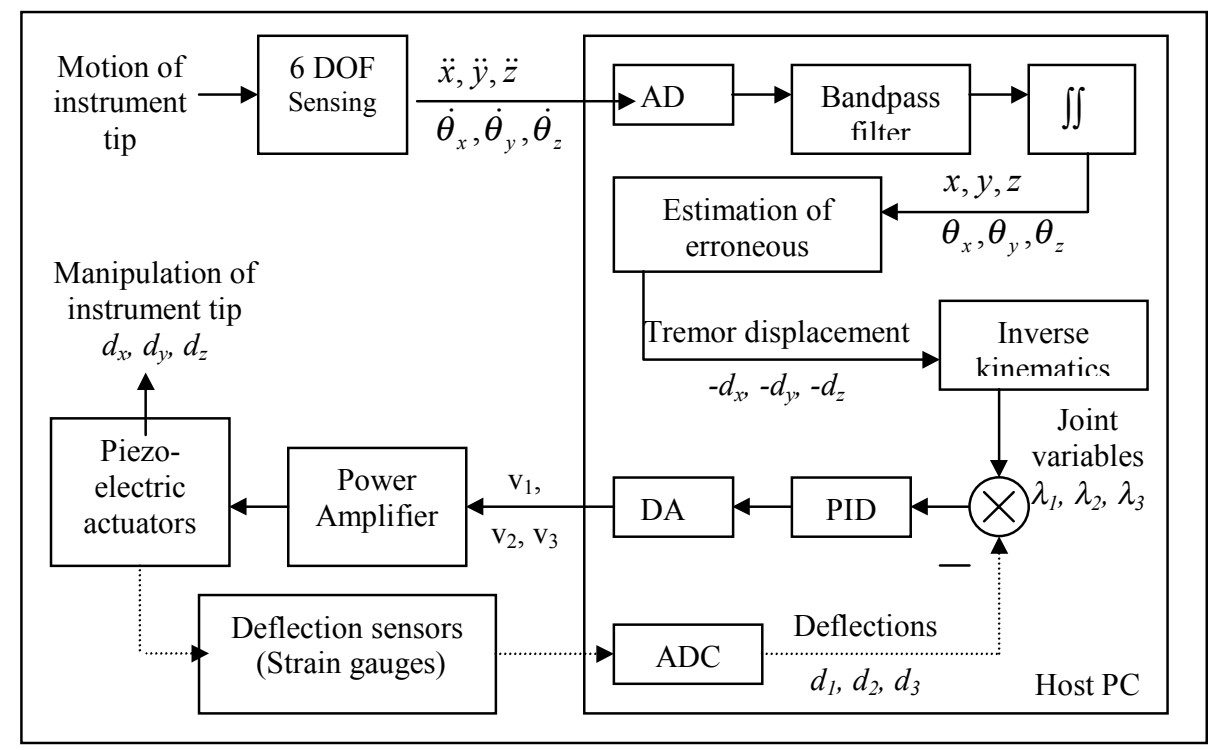

Fig. 2. Block diagram of the Micron system 
The authors' previous work includes development of a motion sensing module for Micron [12], as well as algorithms for online estimation of tremor [13] and undesired non-tremorous components [14] of instrument motion. This work is summarized in Sections 2.3 and 2.4. Section 2.5 presents current work in the design, kinematics, and control of the manipulator for instrument tip deflection.

\subsection{Motion Sensing}

The motion-sensing module is mounted at the back end of the instrument handle, to detect translation and rotation in 6-dof [12], as seen in Figure 1(a). The sensor suite houses six inertial sensors: a CXL02LF3 tri-axial accelerometer (Crossbow Technology, Inc., San Jose, Ca.) and three CG-16D ceramic rate gyros (Tokin Corp., Tokyo). Using the data from these sensors, the three-dimensional (3-D) velocity of the instrument tip is obtained via appropriate kinematic calculations, and then integrated to obtain tip displacement. The module has been shown to estimate tremor-like oscillations $(10 \mathrm{~Hz}, 30 \mu \mathrm{m} \mathrm{p}-\mathrm{p})$ with rms error of approximately $3 \mu \mathrm{m}$. Details of the kinematics and experimental results are presented in [12].

\subsection{Error Estimation for Canceling}

Tremor. Estimation of tremor will be performed by a system based on the weightedfrequency Fourier linear combiner (WFLC) algorithm [13]. The WFLC is an adaptive algorithm that estimates tremor using a dynamic sinusoidal model, estimating its time-varying frequency, amplitude, and phase online. Active canceling of physiological tremor using this algorithm has been demonstrated using a 1-dof instrument prototype. In 25 tests on hand motion recorded from eye surgeons, this technique yielded average rms amplitude reduction of $69 \%$ in the $6-16 \mathrm{~Hz}$ band, and average rms error reduction of $30 \%$ with respect to an off-line estimate of the tremorfree component of motion [13].

Non-tremorous Error. A neural network technique for estimation in real time of non-tremorous erroneous movement has also been developed, using the cascade learning architecture [15], which adjusts not only the values of its weights, but also the number and transfer functions of its hidden nodes. Extended Kalman filtering is used for learning [16]. This technique has been tested in simulation on recordings of vitreoretinal instrument movement, using 100 input nodes (in a tapped delay line), ten hidden nodes, and one output node. These tests resulted in an average rms error reduction of $44 \%$ [14].

\subsection{Manipulator}

Design. The tip of the intraocular shaft may be approximated as a point in Euclidean space. We may disregard changes in orientation of the intraocular shaft, since they will be small in any case, given the small workspace of the manipulator. This reduces the dimension of the configuration space of the manipulator to three, and simplifies the mechanical design and the online computation of inverse kinematics. A parallel manipulator design is best suited to this application because of its rigidity, compactness, and simplicity in design, as compared to a serial mechanism.

Piezoelectric actuators were chosen for their high bandwidth. The TS18-H5-202 piezoelectric stack actuator (Piezo Systems, Inc., Cambridge, Ma.) measures $5 \mathrm{~mm} \mathrm{x}$ 
$5 \mathrm{~mm} \times 18 \mathrm{~mm}$, and deflects to a maximum of about $14.5 \mu \mathrm{m}$ with an applied voltage of $+100 \mathrm{VDC}$. It offers good control linearity, an excellent response time of $50 \mu \mathrm{s}$ and an actuation force of up to $840 \mathrm{~N}$. A range of motion of $100 \mu \mathrm{m}$ or greater has been achieved in each of the three coordinate directions by stacking seven piezoelectric elements to form each actuator. The response time of the piezoelectric actuator ensures the velocities in the joint space are more than adequate to map out the trajectory of the instrument tip in the workspace at the speed needed for canceling of tremor.

Fig.1(b) depicts the intraocular shaft manipulator. The $30 \mathrm{~mm}$ stainless steel intraocular shaft is fixed at the center of the three-legged rigid star. The three legs of the rigid star form the apexes of an equilateral triangle. The rigid star is screwed onto the "flexi-star," which has the exact same shape, by a contact pin at each of its legs. The flexi-star is a flexible thin plate made of ABS 780 thermoplastic. The flexi-star is bolted to the triangular column by three bolts close to its center, which constrains it in the three degrees of freedom that are not being driven, namely, translation in the two coordinates transverse to the long axis of the instrument, and rotation about the long axis.

The stacked piezoelectric actuators are located on the three faces of the triangular column, and sandwiched between the base star and the contact pins. When voltage is applied to the piezoelectric stacks, they expand and push against the contact pins and the base star. This deflects the three overhanging legs of the flexi-star and in turn moves the intraocular shaft on the rigid star. There is a calibration screw at each of the three legs of the base star to compensate for the manufacturing inconsistencies in the length of the piezoelectric actuators. The manipulator system fits within the main housing of the instrument handle, with an interface to the sensor suite at the back end of the handle. The specifications of the manipulator are summarized in Table 1.

Table 1. Specifications of Micron manipulator system.

\begin{tabular}{|l|c|c|c|}
\hline & $x$-axis & $y$-axis & $z$-axis \\
\hline Maximum tip displacement $(\mu \mathrm{m})$ & 560 & 560 & 100 \\
\hline Maximum tip velocity $(\mu \mathrm{m} / \mu \mathrm{s})$ & 11.2 & 11.2 & 2 \\
\hline
\end{tabular}

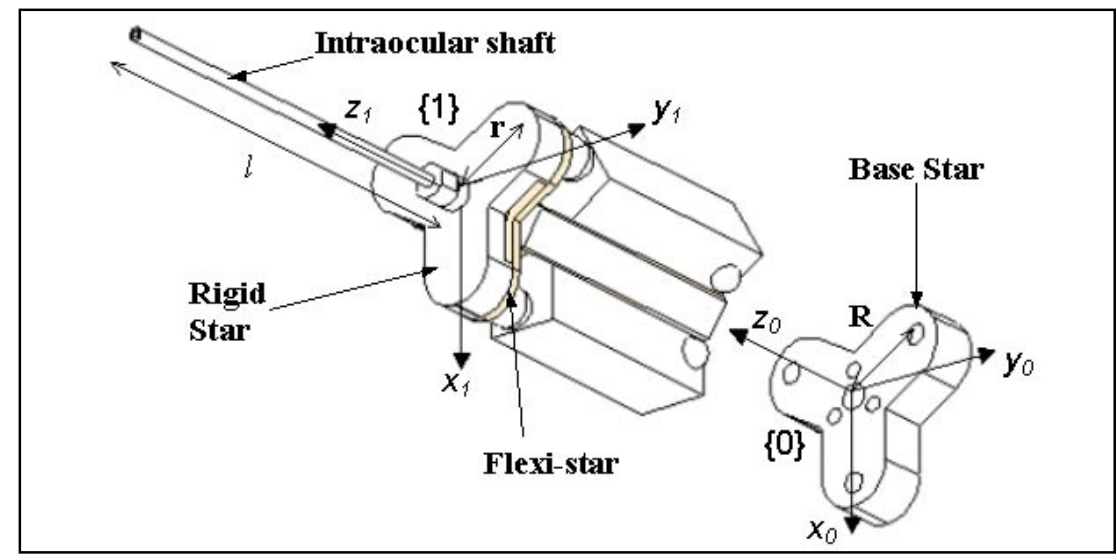

Fig. 3. Kinematic frames of the intraocular shaft manipulator of Micron. 
Kinematics and Control. Since the orientation of the instrument tip in its workspace is unimportant, the dexterous workspace of the manipulator is equivalent to its reachable workspace. Moreover, this type of parallel manipulator is homeomorphic, i.e., it has a one-to-one forward and inverse mapping between its joint space and Euclidean space. It therefore has only boundary singularities and no internal singularities.

The base coordinate system $\{0\}$ is attached to the centroid of the base star, and Frame $\{1\}$ is attached to the centroid of the rigid star such that the $z_{1}$ axis aligns with the intraocular shaft, as shown in Fig. 3. Frame $\{1\}$ has the same orientation as $\{0\}$ and the origin of $\{1\}\left(x_{c}, y_{c}, z_{c}\right)$ has a position of $\left\{0,0, z_{c 0}\right\}$ with respect to $\{0\}$. The value $R$ is the distance between the centroid of base star (origin of $\{0\}$ ) and the vertex of the equilateral triangle form by its three legs. Similarly, the value $r$ is the distance between the centroid of the rigid star (origin of $\{1\}$ ) and the vertex of the equilateral triangle formed by its three legs. In this design, the ratio $\rho=r / R=1$.

Within the small workspace for which the manipulator is intended, the constraints imposed by the design features make its kinematics essentially equivalent to those of Lee and Shah [17]. We define vectors $\boldsymbol{n}, \boldsymbol{o}$, and $\boldsymbol{a}$ to be the directional cosines of the principle axes of $\{1\}$ with respect to those of $\{0\}$.

Let the displacement of the intraocular shaft caused by tremor to be $\left(-d_{x},-d_{y},-d_{z}\right)$. Thus, the canceling displacement of the intraocular shaft $\left(d_{x}, d_{y}, d_{z}\right)$ would be:

$$
\begin{aligned}
& d_{x}=x_{c}+l a_{x} . \\
& d_{y}=y_{c}+l a_{y} . \\
& \quad d_{z}=z_{c}-z_{c 0}+l\left(a_{z}-1\right) .
\end{aligned}
$$

where $l$ is the length of the intraocular shaft. The bolts mounting the flexi-star impose the following constraints:

$$
\begin{aligned}
& n_{y}=o_{x} . \\
& X_{c}=1 / 2 \rho\left(n_{x}-o_{y}\right) . \\
& Y_{c}=-n_{y} \rho .
\end{aligned}
$$

where $X_{c}=x_{c} / R ; Y_{c}=y_{c} / R$.

Let $\lambda_{1}, \lambda_{2}$ and $\lambda_{3}$ be the joint space variables or the extensions of the piezoelectric actuators. With six additional constraints imposed by the orthonormality of $\boldsymbol{n}, \boldsymbol{o}$, and $\boldsymbol{a}$, the system of equations (1)-(6) is then solved for $\lambda_{1}, \lambda_{2}$ and $\lambda_{3}$. The actuator displacements needed for a compensation displacement of $\left(d_{x}, d_{y}, d_{z}\right)$ are then:

$$
\begin{aligned}
& \lambda_{1}=\frac{R}{2} \sqrt{\left(n_{x} \rho+X_{c}-1\right)^{2}+\left(n_{y} \rho+Y_{c}\right)^{2}+\left(n_{z} \rho+Z_{c}\right)^{2}}-z_{c 0} . \\
& \lambda_{2}=\frac{R}{2} \sqrt{\left(-n_{x} \rho+\sqrt{3} o_{x} \rho+2 X_{c}+1\right)^{2}+\left(-n_{y} \rho+\sqrt{3} o_{y} \rho+2 Y_{c}-\sqrt{3}\right)^{2}+\left(-n_{z} \rho+\sqrt{3} o_{z} \rho+2 Z_{c}\right)^{2}}-z_{c 0} . \\
& \lambda_{3}=\frac{R}{2} \sqrt{\left(-n_{x} \rho-\sqrt{3} o_{x} \rho+2 X_{c}+1\right)^{2}+\left(-n_{y} \rho-\sqrt{3} o_{y} \rho+2 Y_{c}+\sqrt{3}\right)^{2}+\left(-n_{z} \rho-\sqrt{3} o_{z} \rho+2 Z_{c}\right)^{2}}-z_{c 0} .
\end{aligned}
$$

where $Z_{c}=z_{c} / R$. Further details on the manipulator kinematics can be found in [14]. 


\section{Manipulator Testing and Results}

\subsection{Testing}

For testing, an infrared LED was mounted on the instrument tip, and its motion was tracked using a specialized optical tracker for micromanipulation [18]. In initial tests, Micron was driven to generate two trajectories:

1. Sinusoidal 1-D motion in the long $(z)$ axis. The target trajectory was $80 \mu \mathrm{m} p-\mathrm{p}$ at $10.2 \mathrm{~Hz}$.

2. A circular motion in the transverse $(x-y)$ plane. The target trajectory had a radius of $70 \mu \mathrm{m}$ and a frequency of $10.2 \mathrm{~Hz}$. Due to the kinematics of the parallel manipulator, tracing this planar figure is actually a 3-dof task in the configuration space, and therefore offers a demonstration of the full 3-dof capabilities of the manipulator.

\subsection{Results}

Fig. 4 shows the results of the first test over 500 data points. The root-mean-square (rms) error for the 1-D trajectory is $2.5 \mu \mathrm{m}$. Fig. 5 presents the results from the 3-dof test over $0.5 \mathrm{~s}$. The rms error with respect to the circular target trajectory is $11.2 \mu \mathrm{m}$.

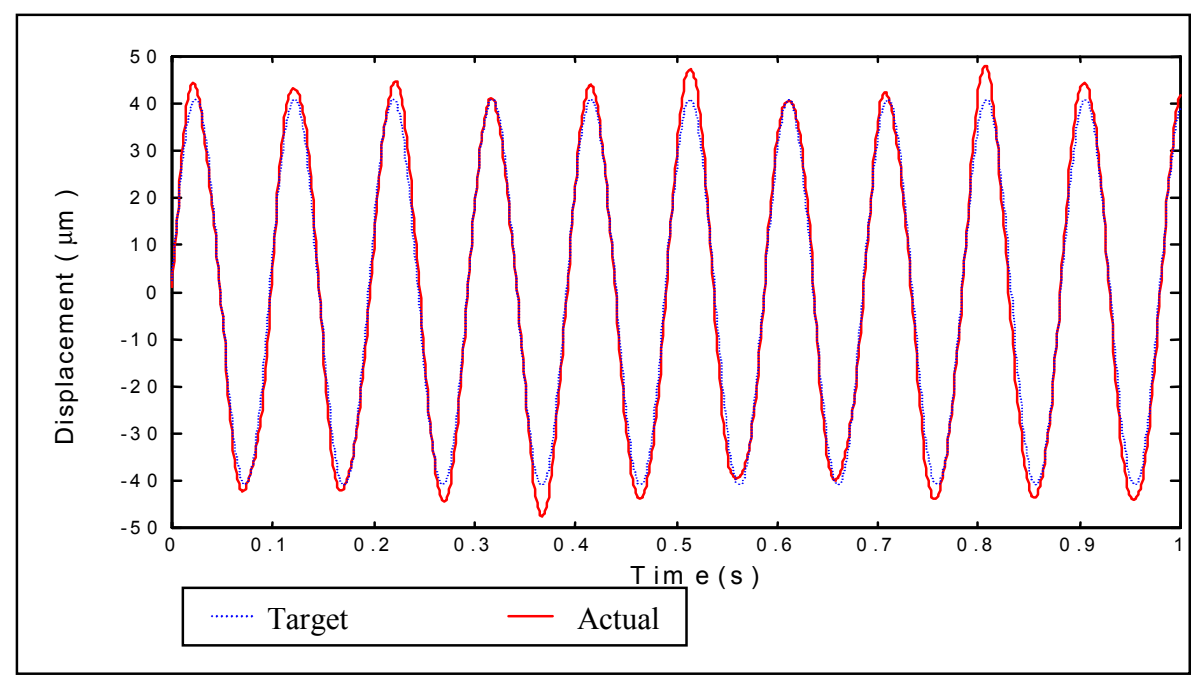

Fig. 4. Open-loop performance of Micron in axial (z-axis) trajectory generation. The dotted line depicts the predicted trajectory, the solid line the actual motion of the instrument tip. 


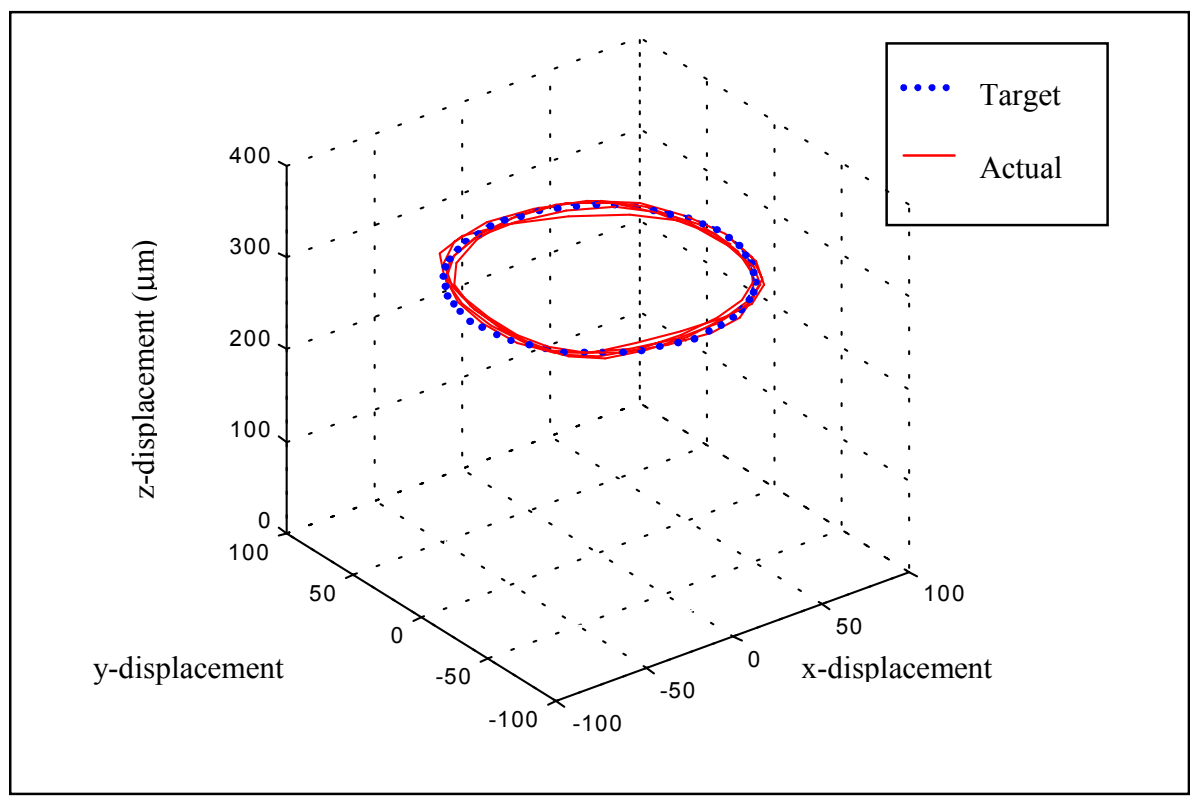

Fig. 5. Open-loop performance of Micron in 3-dof trajectory generation. Though the circle is a planar figure, tracing a circle is actually a 3-dof task in the configuration space, due to the manipulator kinematics. The dotted line depicts the predicted trajectory, the solid line the actual motion of the instrument tip.

\section{Discussion}

The experimental results demonstrate the ability of the manipulator to follow trajectories of the kind necessary to cancel physiological tremor. The piezoelectric actuators exhibit a certain degree of hysteresis, degrading the accuracy. Future work on Micron includes development of closed-loop control, which will minimize this source of error. Experiments will soon be performed in active error compensation, involving all components of Micron: the sensor suite, the error estimation, and the manipulator. In the long term, Micron will be redesigned for lighter weight and smaller size. This can be accomplished by using custom-designed actuators rather than off-the-shelf elements, and by continuing to exploit new developments in MEMS-based inertial sensing.

\section{Conclusion}

The design and preliminary testing of the first prototype of Micron, an active handheld microsurgical instrument for accuracy enhancement, has been presented. It features six inertial sensors and three piezoelectric actuators. The manipulator is able to follow 1-D and 3-D trajectories with rms error of $2.5 \mu \mathrm{m}$ and $11.2 \mu \mathrm{m}$ respectively. 


\section{Acknowledgments}

This work was funded by the Johnson \& Johnson Focused Giving Program. The authors are grateful to Drs. R. Hollis and A. Rizzi for the donation of circuit hardware for the ASAP tracking system.

\section{References}

1. M. Patkin, Ergonomics applied to the practice of microsurgery. Austr. N. Z. J. Surg. 47:320-239, 1977.

2. S. Charles, Dexterity enhancement for surgery. In: R. H. Taylor et al. (eds.): Computer Integrated Surgery: Technology \& Clinical Applications. MIT Press, Cambridge (96) 467-471.

3. W. M. Tang and D. P. Han, "A study of surgical approaches to retinal vascular occlusions." Arch. Ophthalmol. 118:138-43, 2000.

4. J. N. Weiss, "Treatment of central retinal vein occlusion by injection of tissue plasminogen activator into a retinal vein," Am. J. Ophthalmol. 126:142-144, 1998.

5. C. N. Riviere, R. S. Rader, and P. K. Khosla, "Characteristics of hand motion of eye surgeons," Proc. 19th Annu. Conf. IEEE Eng. Med. Biol. Soc., Chicago, 1997.

6. R. C. Harwell and R. L. Ferguson, "Physiologic tremor and microsurgery," Microsurgery, vol. 4, pp. 187-192, 1983.

7. R. J. Elble and W. C. Koller, Tremor. Baltimore: Johns Hopkins, 1990.

8. C. N. Riviere and P. S. Jensen, "A study of instrument motion in retinal microsurgery," submitted to 21st Annu. Conf. IEEE Eng. Med. Biol. Soc., 2000.

9. I. W. Hunter, T. D. Doukoglou, S. R. Lafontaine, P. G. Charette, L. A. Jones, M. A. Sagar, G. D. Mallinson, and P. J. Hunter, "A teleoperated microsurgical robot and associated virtual environment for eye surgery," Presence, 2:265-280, 1993.

10. P. S. Schenker, E. C. Barlow, C. D. Boswell, H. Das, S. Lee, T. R. Ohm, E. D. Paljug, G. Rodriguez, and S.T.2, "Development of a telemanipulator for dexterity enhanced microsurgery," Proc. 2nd Intl. Symp. Med. Robot. Comput. Assist. Surg., pp. 81-88, 1995.

11. R. Taylor, P. Jensen, L. Whitcomb, A. Barnes, R. Kumar, D. Stoianovici, P. Gupta, Z. Wang, E. de Juan, and L. Kavoussi, "A steady-hand robotic system for microsurgical augmentation," in: C. Taylor, A. Colchester (eds.), Medical Image Computing and Computer-Assisted Intervention-MICCAI'99. Springer, Berlin, 1999, pp. 1031-1041.

12. M. Gomez-Blanco, C. Riviere, and P. Khosla, "Sensing hand tremor in a vitreoretinal microsurgical instrument," tech. report CMU-RI-TR-99-39, Robotics Institute, Carnegie Mellon University, Pittsburgh, Pa., 1999.

13. C. N. Riviere, R. S. Rader, and N. V. Thakor, "Adaptive canceling of physiological tremor for improved precision in microsurgery,” IEEE Trans. Biomed. Eng., vol. 45, pp. 839-846, 1998.

14. C. N. Riviere and P. K. Khosla, "Augmenting the human-machine interface: improving manual accuracy,” Proc. IEEE Intl. Conf. Robot. Autom., Albuquerque, N.M., Apr. 20-25, 1997.

15. M. C. Nechyba and Y. Xu, "Learning and transfer of human real-time control strategies," J. Adv. Computational Intell. 1(2):137-54, 1997.

16. G. V. Puskorius and L. A. Feldkamp, "Decoupled extended Kalman filter training of feedforward layered networks," Proc. Intl. Joint Conf. Neural Networks, 1:771-7, 1991.

17. K.-M. Lee and D. K. Shah, "Kinematic analysis of a three-degrees-of-freedom in-parallel actuated manipulator,” IEEE Trans. Robot. Autom., 4:354-360, 1988.

18. C. N. Riviere and P. K. Khosla, Microscale tracking of surgical instrument motion. In: C. Taylor, A. Colchester (eds.), Medical Image Computing and Computer-Assisted Intervention--MICCAI'99. Springer, Berlin, 1999, pp. 1080-1087. 